\title{
Ações neuroprotetoras da vitamina $C$ no corpo estriado de ratos após convulsões induzidas pela pilocarpina
}

\author{
Neuroprotective actions of vitamin C in rat striatum after pilocarpine-induced seizures
}

\author{
Rivelilson Mendes de Freitas'1, Adriana da Rocha Tomé2 2 \\ 1 Departamento de Farmacologia da Universidade Federal do Piauí (UFPI), Picos, PI. \\ 2 Universidade Estadual do Ceará (UECE), Campus do Itaperi, Fortaleza, CE.
}

Recebido: 13/8/2009 - Aceito: 29/9/2009

\begin{abstract}
Resumo
Contexto: As convulsões podem produzir danos neuronais em diversas áreas e, especialmente, nas estruturas límbicas. Objetivos: O objetivo do presente trabalho foi estudar os efeitos neuroprotetores da vitamina $\mathrm{C}$ nas alterações histopatológicas observadas no corpo estriado de ratos convulsivos. Material e métodos: Foram utilizados ratos Wistar adultos. Os animais foram divididos em quatro grupos. O primeiro grupo foi tratado com salina 0,9\% (grupo controle) e o segundo, com pilocarpina (400 mg/kg, grupo P400). Já o terceiro e o quarto grupo foram tratados com vitamina C ( $250 \mathrm{mg} / \mathrm{kg}$ ), e, 30 minutos depois, receberam P400 (grupo VIT C + P400) ou solução salina $0,9 \%$ (grupo VIT C), respectivamente. Após os tratamentos, todos os grupos foram observados por 24 horas e, em seguida, sacrificados e seus cérebros removidos para as análises histopatológicas. Resultados: O grupo P400 apresentou convulsões que progrediram para o estado epiléptico em $75 \%$ dos animais. O pré-tratamento com vitamina C produziu uma redução de $35 \%$ nesse índice. Os grupos P400 e VIT C + P400 apresentaram $80 \%$ e $20 \%$ de animais com lesão cerebral, respectivamente. No corpo estriado dos animais do grupo P400, houve um comprometimento de $50 \%$. Por sua vez, na região estriatal dos animais do grupo VIT C + P400 foi vista uma redução de 40\% nesse comprometimento. Discussão: As convulsões induzidas pela pilocarpina são instaladas pelo sistema colinérgico e propagadas pela produção de radicais livres e pelo sistema glutamatérgico, resultado no desenvolvimento de dano cerebral. As drogas antioxidantes podem apresentar um potencial terapêutico para pacientes epilépticos na proteção contra as lesões cerebrais por meio da remoção desses radicais livres formados. Acredita-se, assim, que a vitamina $\mathrm{C}$ pode influenciar a epileptogênese e promover ações neuroprotetoras durante as convulsões.
\end{abstract}

Freitas RM, Tomé AR / Rev Psiq Clín. 2010;37(3):105-8

Palavras-chave: Convulsão, lesão cerebral, corpo estriado, pilocarpina, ácido ascórbico.

\begin{abstract}
Background: Seizures may produce neuronal damage in several areas and especially in limbic structures. Objectives: This study aimed to evaluate the neuroprotective effects of vitamin $\mathrm{C}$ in the histopathological changes observed in rat striatum after seizures. Material and methods: Healthy Wistar rats were divided into four groups. The first group was treated with $0.9 \%$ saline (control group) and the second one with pilocarpine ( $400 \mathrm{mg} / \mathrm{kg}$, P400 group). Third and fourth groups were treated with vitamin C ( $250 \mathrm{mg} / \mathrm{kg}$ ), 30 minutes before receiving P400 (P400 + VIT C group) or $0.9 \%$ saline (VIT C group), respectively. After the treatments, all groups were observed for 24 hours, sacrificed and dissected out to remove their brains for histopathological analysis. Results: The group P400 presented seizures that progressed to status epilepticus in $75 \%$ of the animals. Pretreatment with vitamin C produced a $35 \%$ reduction in this index. P400 and P400 + VIT C groups revealed $80 \%$ and $20 \%$ of animals with brain injury, respectively. In P400 group, lesion severity of the striatum was $50 \%$. In turn, in striatal region of animals treated with P400 + VIT C group, we detected a reduction of $40 \%$ in the severity degree. Discussion: Pilocarpine-induced seizures are installed by the cholinergic system and propagated by free radicals and by glutamatergic system, leading to brain damage. The antioxidant drugs may have therapeutic potential for epileptic patients to protect against brain injure through removing free radicals produced, suggesting that vitamin C may influence epileptogenesis and promote neuroprotective actions during seizures.
\end{abstract}

Freitas RM, Tomé AR / Rev Psiq Clín. 2010;37(3):105-8

Keywords: Seizures, neuronal damage, striatum, pilocarpine, ascorbic acid.

\section{Introdução}

A vitamina C (ácido ascórbico) é um componente essencial envolvido em várias funções biológicas no organismo humano. Estudos mostram que a diminuição de sua concentração pode produzir inúmeras patologias, incluindo epilepsia ${ }^{1}$. O ácido ascórbico também participa de uma variedade de reações enzimáticas, tais como na síntese de catecolaminas e de colágeno ${ }^{2}$. A literatura relata que existem altas concentrações desse composto antioxidante no sistema nervoso central (SNC), sugerindo um papel importante deste durante os processos de remoção de espécies reativas derivadas do oxigênio (EROS) nos compartimentos intra e extracelular neuronal, atribuindo-se a este a capacidade de participar em inúmeros processos fisiopatológicos ${ }^{3}$.
Dados recentes demonstraram que o ácido ascórbico participa como um importante neuromodulador da transmissão glutamatérgica no SNC, contribuindo para a redução da excitotoxicidade mediada pelo glutamato nas terminações nervosas ${ }^{4}$. O metabolismo alterado do glutamato e a disfunção das moléculas transportadoras glutamatérgicas podem facilitar a estimulação dos receptores glutamatérgicos, induzindo um aumento na concentração intracelular de cálcio ${ }^{5}$, que, por sua vez, facilita a ativação de várias enzimas de importância fisiopatológica durante as convulsões.

O glutamato parece desencadear a liberação de ascorbato em astrócitos ${ }^{6}$. Existe evidência de que a perda de moléculas transportadoras de glutamato nessas células neuronais pode contribuir para a alteração das funções cerebrais ${ }^{5,7}$. Além disso, a alteração de transportadores de glutamato no córtex cerebral pode exacerbar a 
lesão cerebral isquêmica. Essa ação neurotóxica pode ser aumentada pelo estresse oxidativo ${ }^{8}$. Sabe-se que o sistema glutamatérgico tem uma importante participação na instalação e na propagação e é responsável pelas lesões cerebrais induzidas pelas convulsões ${ }^{9}$. Estudos anteriores demonstram que a administração de pilocarpina induz o desenvolvimento de convulsões, estado epiléptico e lesão cerebral em roedores $^{9-11}$. O início das convulsões pode ser bloqueado por drogas anticolinérgicas sugerindo que a ativação do receptor colinérgico muscarínico é responsável pela instalação do processo convulsivo. Por sua vez, o bloqueio de receptores glutamatérgicos impede sua propagação e o desenvolvimento de dano cerebral4,11,12.

Fazendo uso do modelo de convulsões induzidas pela pilocarpina, tem sido estudadas a severidade e as áreas cerebrais afetadas durante o desenvolvimento das lesões cerebrais observadas nesse modelo. No entanto, os efeitos de drogas antioxidantes durante a evolução temporal dessas lesões cerebrais ainda não foram completamente estabelecidos. De maneira geral, as convulsões límbicas produzem danos neuronais em diversas áreas, causando perda neuronal em várias estruturas, a saber: hipocampo, corpo estriado, amígdala, córtex piriforme, córtex entorrinal, córtex frontal, tálamo e substância negra ${ }^{13,14}$.

Dessa forma, por meio do modelo de convulsão induzida por pilocarpina, decidiu-se estudar os efeitos neuroprotetores da vitamina C nas alterações histopatológicas observadas no corpo estriado de ratos convulsivos, uma vez que essa área tem sido sugerida como responsável pela propagação e/ou manutenção da atividade convulsiva induzida pela pilocarpina ${ }^{15}$.

\section{Material e métodos}

Foram utilizados ratos Wistar adultos (2 meses de idade; machos; $\mathrm{n}=38$ ), com peso variando entre 250 e $280 \mathrm{~g}$, provenientes do Biotério Central do Departamento de Fisiologia e Farmacologia, da Faculdade Católica Rainha do Sertão. Durante todos os experimentos, os animais foram mantidos em gaiolas de acrílico de $30 \times 30 \times 17 \mathrm{~cm}^{3}$, onde foram observados durante 24 horas com no máximo seis animais, em condições ambientais semelhantes, com ciclo claro/escuro alternado de 12 horas (07:00 a.m.), recebendo ração padrão tipo Purina e água $a d$ libitum. Os experimentos foram realizados de acordo com o guia de cuidados e usos de animais de laboratório do Departamento de Saúde e Serviços Humanos dos Estados Unidos da América (EUA).

Os animais foram divididos em quatro grupos. O primeiro grupo foi tratado com solução salina $0,9 \%$ (via intraperitoneal (i.p.), $\mathrm{n}=$ 6, grupo controle). O segundo grupo foi tratado com pilocarpina na dose de $400 \mathrm{mg} / \mathrm{kg}$ (i.p., $\mathrm{n}=12 ; \mathrm{P} 400$ ). Já o terceiro e quarto grupo foram tratados com vitamina $\mathrm{C}$ na dose de $250 \mathrm{mg} / \mathrm{kg}$ por via intraperitoneal, e 30 minutos depois receberam pilocarpina ( $400 \mathrm{mg} / \mathrm{kg}$, i.p., $\mathrm{n}=6$, grupo VIT C + P400) ou solução salina $0,9 \%$ (i.p., $\mathrm{n}=6$, grupo VIT C), respectivamente. Após os tratamentos, os grupos foram colocados em gaiolas para observação dos seguintes parâmetros (sinais colinérgicos periféricos [SCP], tremores, movimentos estereotipados [ME], convulsões, estado epiléptico [EP] e taxa de mortalidade). O grupo P400 foi constituído pelos animais que apresentaram convulsão, estado epiléptico e que sobreviveram ao período de 24 horas de observação.

Após o período de observação de 24 horas, todos os grupos foram sacrificados por decapitação. Seus cérebros foram removidos e fixados em formalina a $10 \%$ por 72 horas para a realização das análises histopatológicas. Cortes sagitais, feitos em intervalos de $1 \mathrm{~mm}$, foram obtidos a partir de um corte inicial próximo aos corpos mamilares. Para o estudo microscópico, secções de $10 \mu$ foram feitas, coradas em hematoxilina-eosina (HE), e analisadas com auxílio de um microscópio óptico. As áreas cerebrais foram observadas e classificadas de acordo com as descrições do Atlas de Paxinos e Watson ${ }^{16}$. O grau de lesão foi expresso por meio de uma escala percentual de 0 (nenhum) a 100 (total) para cada corpo estriado analisado de acordo com o método descrito anteriormente ${ }^{17,18}$. A lesão cerebral foi definida pela presença de pelo menos $50 \%$ de alteração histopatológica em cada corpo estriado analisado.
Para a análise estatística, os resultados não paramétricos (percentagens) foram analisados pelo programa GraphPad Prism versão 3.00 para Windows, GraphPad software, San Diego Califórnia, EUA. Copyright (c) 1994-1999 por GraphPad software utilizando o teste do qui-quadrado. As diferenças foram consideradas estatisticamente significativas a partir de $\mathrm{p}<0,05$.

\section{Resultados}

A tabela 1 mostra que todos os animais tratados com pilocarpina (grupo P400, $\mathrm{n}=24$ ) apresentaram SCP, ME e tremores. As convulsões ocorreram em $75 \%$ (18) dos animais, que progrediram para o estado epiléptico também em 75\% (18) dos animais. A taxa de mortalidade desse grupo corresponde a $63 \%$ (15).

Tabela 1. Efeito de pré-tratamento com vitamina $C$ em ratos durante as convulsões límbicas

\begin{tabular}{l|c|c|c|c|c|c}
\hline Grupos & \multicolumn{1}{c}{$\begin{array}{c}\text { SCP } \\
(\%)\end{array}$} & $\begin{array}{c}\text { ME } \\
(\%)\end{array}$ & $\begin{array}{c}\text { Tremores } \\
(\%)\end{array}$ & $\begin{array}{c}\text { Convulsões } \\
(\%)\end{array}$ & $\begin{array}{c}\text { EP } \\
(\%)\end{array}$ & $\begin{array}{c}\text { Taxa de } \\
\text { mortalidade } \\
(\%)\end{array}$ \\
\hline P400 & 100 & 100 & 100 & 75 & 75 & 63 \\
\hline VIT C + P400 & 100 & 100 & 100 & $40^{\mathrm{a}}$ & $40^{\mathrm{a}}$ & $20^{\mathrm{a}}$ \\
\hline
\end{tabular}

a $p<0,05$, quando comparado ao grupo P400. Teste do Qui-quadrado. SCP: sinais colinérgicos periféricos; ME: movimentos estereotipados; EP: estado epiléptico.

O grupo P400 foi tratado com pilocarpina $(400 \mathrm{mg} / \mathrm{kg}$, i.p., $\mathrm{n}=24)$. O grupo VIT C + P400 foi pré-tratado com vitamina C (250 $\mathrm{mg} / \mathrm{kg}$, i.p.) e 30 minutos após recebeu pilocarpina ( $400 \mathrm{mg} / \mathrm{kg}$, i.p., $\mathrm{n}=10$ ). Após o tratamento com pilocarpina, foram observados durante 24 horas para a determinação dos SCP, ME, tremores, convulsões, EP e taxa de mortalidade. Os resultados dos estudos comportamentais foram expressos em percentagem.

Os dados apresentados na tabela 1 sugerem que os efeitos produzidos pela estimulação colinérgica periférica não são bloqueados pela vitamina C. O pré-tratamento com vitamina C (grupo VIT C + P400) não interfere em SCP, ME e tremores induzidos pela pilocarpina. No entanto, produziu um aumento na latência da primeira convulsão e na latência da instalação do estado epiléptico (dados não mostrados). No mesmo grupo, notou-se uma diminuição de $35 \%$ no número de animais que convulsionam e que evoluem para o EP $(\mathrm{p}<0,05)$. Foi verificada também uma redução na taxa de mortalidade de $43 \%$ $(\mathrm{p}<0,05)$. Os grupos tratados somente com solução salina $0,9 \%$ (grupo controle) ou vitamina C (grupo VIT C) não apresentaram nenhuma alteração comportamental (Tabela 1).

A figura 1 mostra uma alta percentagem de animais com lesão cerebral no grupo P400. Por sua vez, nos animais que foram prétratados com vitamina C, 30 minutos antes da administração de pilocarpina, houve redução no número de animais com lesão cerebral e, daqueles que apresentaram convulsão e EP, detectou-se alteração histopatológica no corpo estriado em apenas dois animais. Também foi detecta uma redução de $40 \%$ no grau de comprometimento do corpo estriado no grupo VIT C + P400, quando comparado ao grupo $\mathrm{P} 400$. Os grupos tratados somente com solução salina $0,9 \%$ (grupo controle) ou vitamina C (grupo VIT C) não apresentaram nenhuma alteração histopatológica (Figura 1).

\section{Discussão}

No presente estudo, demonstrou-se que a vitamina $\mathrm{C}$ reduz o número de animais que apresentam convulsões e EP induzido por pilocarpina e que também essa droga antioxidante aumenta o índice de sobrevivência dos animais. $\mathrm{O}$ ácido ascórbico tem a capacidade de reparar a lesão neuronal, juntamente com outras enzimas antioxidantes (glutationa peroxidase e glutationa redutase) ${ }^{19}$, sugerindo, assim, um papel neuroprotetor para a vitamina C.

Dados anteriores evidenciaram uma supressão da atividade do transportador de glutamato em astrócitos de ratos pelo peroxinitrito, contribuindo para o acúmulo de glutamato e, consequentemente, 


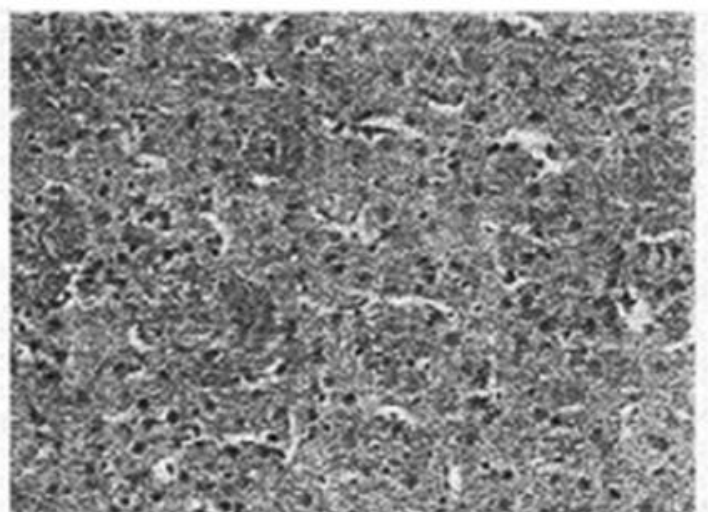

A

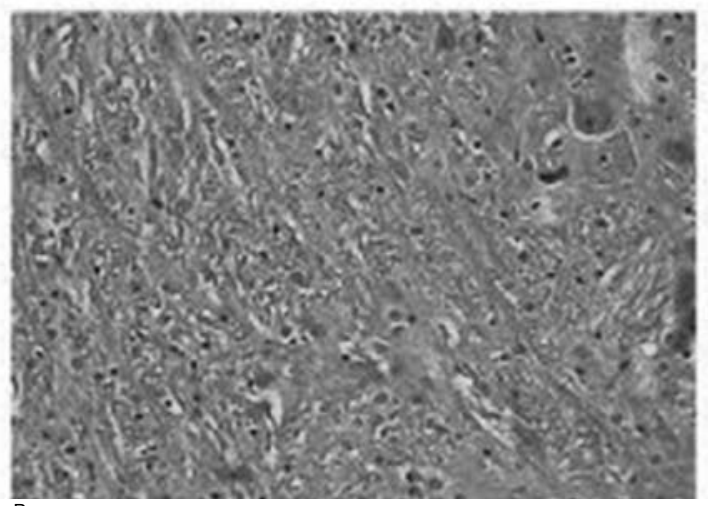

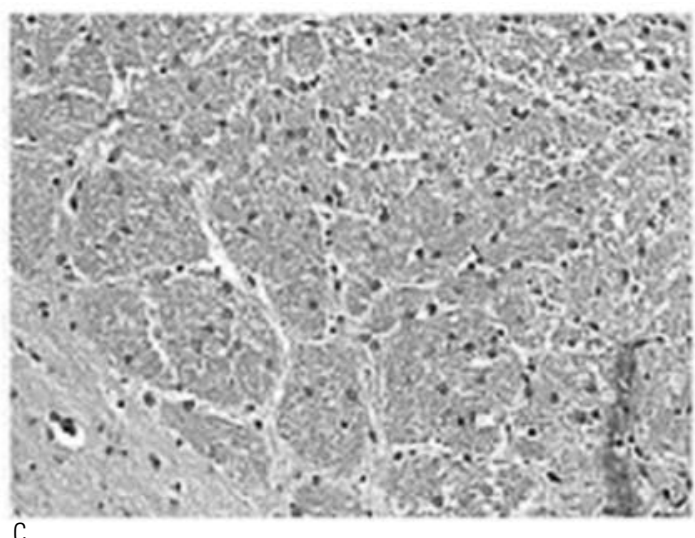

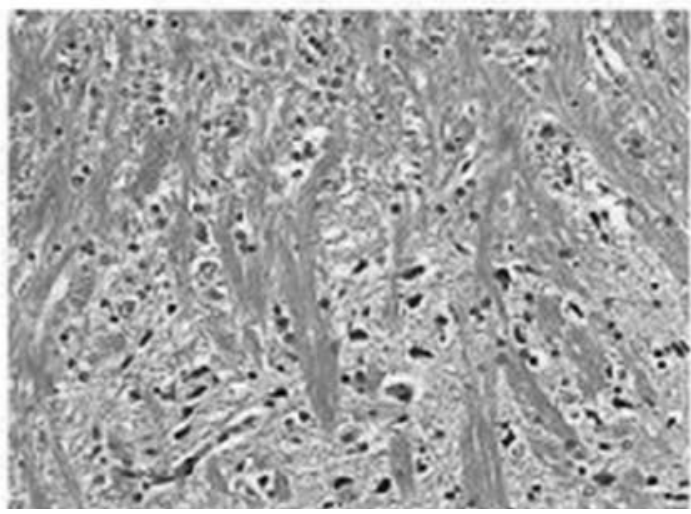

D

Figura 1. Efeitos do pré-tratamento com vitamina $C$ no dano cerebral induzido pelas convulsões.

A - Ausência de alterações histopatológicas no corpo estriado de ratos adultos observados por 24 horas após administração de salina (NaCl 0,9\%, i.p.; $\mathrm{n}=6$, controle) (Hematoxilina-Eosina (HE) - X40); B - Ausência de alterações histopatológicas no corpo estriado de ratos adultos observados por 24 horas após administração de vitamina C (250 mg/kg, i.p.; $\mathrm{n}=6$, VIT C) (HE X40); C - Perda neuronal, gliose, atrofia e degeneração no corpo estriado de ratos adultos que apresentaram convulsão, estado epiléptico e que foram sacrificados 24 horas após a administração de P400 (400 mg/kg, i.p., $\mathrm{n}=4$ ) (HE X40); D - Ausência de alterações histopatológicas no corpo estriado de ratos adultos pré-tratados com vitamina C (250 mg/kg, i.p.) e que após 30 minutos receberam pilocarpina (400 mg/kg, i.p., $n=5$, VIT C + P400) (HE X40).

a propagação das convulsões e a produção de dano cerebral ${ }^{20}$. A vitamina $\mathrm{C}$ é um agente antioxidante que participa do sistema da defesa celular contra lesões induzidas por radicais livres e produz alterações benéficas nos parâmetros comportamentais induzidos pelas convulsões. Os resultados do presente estudo concordam com os dados anteriormente descritos na literatura sobre o papel neuroprotetor da vitamina $\mathrm{C}$ durante as convulsões e EP induzido pela pilocarpina ${ }^{21,22}$. Entretanto, ainda não foram estabelecidas as ações da vitamina $\mathrm{C}$ na proteção contra as lesões cerebrais induzidas pelas convulsões.

A vitamina $C$ contribui para a formação dos tecidos ósseos $\mathrm{e}$ cartilaginosos. A sua deficiência pode induzir deformidades ósseas, hemorragias e anorexia; além disso, facilita o desenvolvimento do processo oxidativo ${ }^{23}$ e retarda o processo de cicatrização ${ }^{24}$. O interesse pelo papel dessa vitamina decorre da constatação de que as adaptações metabólicas verificadas no processo convulsivo conduzem a aumento da taxa metabólica basal, no consumo de oxigênio e na formação de radicais livres. O ácido ascórbico é um importante antioxidante em seres humanos e vários estudos demonstraram redução na concentração plasmática de vitamina $\mathrm{C}$ durante o desenvolvimento das doenças neurodegenerativas, entretanto, o significado dessas alterações durante o estabelecimento das convulsões ainda não está completamente esclarecido.

O modelo de epilepsia induzido pela pilocarpina tem sido extensivamente analisado quanto às mudanças histopatológicas. As alterações observadas no corpo estriado reproduzem muitas dessas alterações observadas em epilépticos, incluindo perda neu- ronal e remoção do tecido de sustentação do $\mathrm{SNC}^{6,25,26}$. A literatura registra danos morfológicos em diversas estruturas cerebrais após convulsão ${ }^{27,28}$. As principais alterações são o alargamento dos ventrículos, a deformação do giro dentado com moderada dispersão das células granulares e a perda neuronal em diversas áreas (hipocampo, córtex cerebral, corpo estriado, córtex piriforme, amígdala, tálamo, núcleo subtalâmico, corpo caloso, córtex motor e área septal).

Esses danos neuronais também foram observados no hipocampo, amígdala, córtex piriforme, córtex entorrinal, septum lateral, tálamo, neocórtex e substância negra de ratos convulsivos ${ }^{13,14}$. Neste trabalho, vários animais foram distribuídos para o estudo histopatológico, sendo detectadas lesão cerebral, vacuolização e gliose no grupo P400. Esses dados sugerem a participação dos sistemas colinérgicos e oxidantes cerebrais nesse processo. No presente estudo, as estruturas cerebrais mais afetadas com a administração de pilocarpina foram, em ordem decrescente de severidade da lesão, o hipocampo, o córtex frontoparietal e entorrinal, seguidos do córtex piriforme, corpo estriado e substância negra.

Neste estudo, demonstrou-se alta percentagem de animais com lesão cerebral após administração de pilocarpina. Por sua vez, os animais pré-tratados com vitamina C, 30 minutos antes da administração de pilocarpina, apresentaram menor número de animais com lesão cerebral. Além disso, naqueles animais que apresentaram convulsão e EP, foi detectada alteração histopatológica no corpo estriado em apenas dois deles, sugerindo, assim, que as lesões decorrentes do processo convulsivo podem ocorrer em consequência da produção de radicais livres. Portanto, esses resultados indicam que o uso de 
drogas antioxidantes pode apresentar ação neuroprotetora durante o tratamento das convulsões em humanos.

O corpo estriado foi descrito como sendo responsável pela propagação da atividade convulsiva induzida pela pilocarpina. Estudos realizados com drogas antioxidantes demonstraram menor índice de lesões estriatais em animais epilépticos. Essas drogas podem ser capazes de produzir efeito antiepileptogênico no modelo da epilepsia do lobo temporal que apresenta alterações histopatológicas semelhantes às de humanos. Esses resultados sugerem uma possível interação entre o sistema colinérgico e os sistemas antioxidantes cerebrais. E, ainda, mostram que a remoção de radicais livres pode ser essencial na prevenção das lesões em algumas áreas cerebrais; no entanto, esses agentes antioxidantes não são capazes de impedir a instalação e/ou cessar a manutenção da atividade convulsiva ${ }^{14,21}$.

As mudanças histopatológicas no corpo estriado reproduzem muitas alterações vistas na epilepsia do lobo temporal em humanos, incluindo perda neuronal e remoção do tecido de sustentação do $\mathrm{SNC}^{25}$. Os dados do presente estudo concordam com os anteriormente descritos, uma vez que foram observadas degeneração neuronal, gliose, atrofia e vacuolização no corpo estriado dos ratos convulsivos. Além disso, foi evidenciada a ação neuroprotetora da vitamina $\mathrm{C}$ nessas lesões por meio da diminuição do número de animais que apresentam dano cerebral e alterações histopatológicas (Figura 1).

Todos os grupos foram observados durante 24 horas e, em seguida, sacrificados para remoção dos cérebros e dissecação do corpo estriado para realização dos estudos histopatológicos. O grau de lesão foi expresso por meio de uma escala percentual de 0 (nenhum) a 100 (total) para cada corpo estriado examinado. Os animais foram definidos como tendo lesão cerebral quando havia pelo menos $50 \%$ de alteração no corpo estriado.

As convulsões induzidas pela pilocarpina são instaladas pelo sistema colinérgico, enquanto a propagação e o desenvolvimento do dano cerebral devem-se ao aumento da produção de EROS e pela estimulação do sistema glutamatérgico. As drogas antioxidantes podem apresentar um potencial terapêutico para pacientes epilépticos na proteção contra as lesões cerebrais durante a atividade epiléptica, provavelmente, por meio da remoção de radicais livres, pelas mudanças nas propriedades da membrana celular e, ainda, pela estabilização das funções sinápticas neuronais. Os dados do presente estudo sugerem que a vitamina $C$ pode influenciar a epileptogênese e promover ações anticonvulsivantes e neuroprotetoras durante as convulsões. Entretanto, novos estudos devem ser realizados no intuito de fortalecer os mecanismos de ação da vitamina $\mathrm{C}$ que justifiquem seu uso no tratamento clínico de pacientes epilépticos.

\section{Agradecimentos}

Este trabalho foi financiado pelo Conselho Nacional de Pesquisa (CNPq). RMF é bolsista de produtividade do CNPq. Gostaríamos de agradecer a Stênio Gardel Maia pela assistência técnica.

\section{Referências}

1. Ten Cate AR. Oral histology: development, structure and function. St. Louis: Mosby; 1998.

2. Padh H. Vitamin C: never insights into its biochemical functions. Nutr Rev. 1991;49:65-70.3.

3. Agus DB, Gambhir SS, Pardridge WM, Spielholz C, Baselga J, Vera JC, et al. Vitamin $\mathrm{C}$ crosses the blood-brain barrier in the oxidized form through the glucose transporters. J Clin Invest. 1997;100:2842-8.

4. Jope RS, Morisett RA, Snead OC. Characterization of lithium potentiation of pilocarpine-induced status epilepticus in rats. Exp Neurol. 1986;91:471-80.

5. Rothstein JD. Excitotoxic mechanisms in the pathogenesis of amyotrophic lateral sclerosis. Adv Neurol. 1995;68:7-20.

6. Mello LEAM, Del Bel EA, Gomes ELT, Oliveira JAC, Wakamatsu H, Cairasco NG. Neuroethological and morphological (Neo-Timm staining) correlates of limbic recruitment during the development of audiogenic kindling in seizures susceptible Wistar rats. Epilepsy Res. 1996;26:177-92.
7. Revuelta M, Romero-Ramos M, Venero JL, Millan F, Machado A, Cano J Less induced 1-methyl-4-phenylpyridinium ion neurotoxicity on striatal slices from guinea-pigs fed with a vitamin C-deficient diet. Neuroscience. 1997;77:167-74.

8. Lai YJ, Shen EY, Pan WH. Effects of ascorbate in microdialysis perfusion medium on the extracellular basal concentration of glutamate in rat's striatum. Neurosci Lett. 2000;279:145-8.

9. Turski L, Ikonomidou C, Turski WA, Bortolotto ZA, Cavalheiro EA. Cholinergic mechanisms and epileptogenesis. The seizures induced by pilocarpine: a novel experimental model of intractable epilepsy. Synapse. 1989;3:154-71.

10. Freitas RM, Viana GSB, Fonteles MMF. Níveis dos neurotransmissores estriatais durante o estado epiléptico. Rev Psiq Clín. 2003;30:76-9.

11. Marinho MMF, Sousa FCF, Bruin VMS, Aguiar LMV, Pinho RSN, Viana GSB. Inhibitory action of a calcium channel blocker (nimodipine) on seizures and brain damage induced by pilocarpine and lithium-pilocarpine in rats. Neurosci Lett. 1997;235:13-6.

12. Morrisett RA, Jope RS, Snead OC. Effects of dugs on the initiation and maintenance of status eplepticus induced by administration of pilocarpine to lithium-pretread rats. Exp Neurol. 1987a; 97:193-200.

13. Clifford DB, Olney JW, Maniotis A, Collins RC, Zorumski CF. The functional anatomy and pathology of lithium-pilocarpine and high-dose pilocarpine seizures. Neuroscience. 1987;23:953-68.

14. Honchar MP, Olney JW, Sherman WR. Systemic cholinergic agents induce seizures and brain damage in lithium-treated rats. Science. 1983a;220:323-5.

15. Turski WA, Cavalheiro EA, Schwartz M, Czuczwar SJ, Kleinrok Z, Turski L. Limbic seizures produced by pilocarpine in rats: a behavioural, electroencephalographic and neuropathological study. Behav Brain Res. 1983a;9:315-35.

16. Paxinos G, Watson C. The rat brain in stereotaxie coordenates. New York: Academic Press; 1986.

17. Bureau YRJ, Peredery O, Persinger MA. Concordance of quantitative damage within the diencephalon and telencephalon following systemic pilocarpine $(380 \mathrm{mg} / \mathrm{kg})$ or lithium $(3 \mathrm{mEq} / \mathrm{kg}) /$ pilocarpine $(30 \mathrm{mg} / \mathrm{kg})$ induced seizures. Brain Res. 1994;648:265-9.

18. Ferreira ALA, Matsubara LS. Radicais livres: conceitos, doenças relacionadas, sistema de defesa e estresse oxidativo. Rev Assoc Med Brasil. 1997;4:61-8.

19. Bano S, Parihar MS. Reduction of lipid peroxidation in different brain regions by a combination of alpha-tocopherol and ascorbic acid. J Neural Transm. 1997;104:1277-86.

20. Barros DO, Xavier SML, Barbosa CO, Silva RF, Freitas RLM, Maia FD, et al. Effects of the vitamin $\mathrm{E}$ in catalase activities in hippocampus after status epilepticus induced by pilocarpine in Wistar rats. Neurosci Lett. 2007;416:227-30.

21. Santos LFL, Freitas RLM, Xavier SML, Saldanha GB, Freitas RM. Neuroprotective actions of vitamin $\mathrm{C}$ related to decreased lipid peroxidation and increased catalase activity in adult rats after pilocarpine-induced seizures. Pharm Biochemd Behav. 2008;89:1-5.

22. Xavier SML, Barbosa CO, Barros DO, Silva RF, Oliveira AA, Freitas RM. Vitamin C antioxidant in hippocampus of adult Wistar rats after seizures and status epilepticus induced by pilocarpine. Neurosci Lett. 2007;420:76-9.

23. Wang X, Kim KW, Bai SC, Hud MD, Cho BY. Effects of the different levels of dietary vitamin $\mathrm{C}$ on growth and tissue ascorbic acid changes in parrot fish (Oplegnathus fasciatus). Aquaculture. 2003;215:203-11.

24. Moraes JRE, Freitas JB, Bozzo FR, Moraes FR, Martins ML. A suplementação alimentar com vitamina $\mathrm{C}$ acelera a evolução do processo cicatricial em Piaractus mesopotamicus. Boletim do Instituto de Pesca. 2003;29:57-60

25. Holmes GL, Cha BH, Akman C, Silveira DC, Liu X. Spontaneous recurrent seizure following status epilepticus enhances dentate gyrus neurogenesis. Brain Dev. 2004;26:394-7.

26. Marchetti RL, Castro AWP, Kurcgant D, Cremonese E, Neto JG. Transtornos mentais associados à epilepsia. Rev Psiq Clín. 2005;32:170-82.

27. Devi PU, Manocha A, Vohora D. Seizures, antiepileptics, antioxidants and oxidative stress: an insight for researchers. Expert Opin Pharmacother. 2008;9:3169-77.

28. Kok AB. Ascorbate availability and neurodegeneration in amyotrophic lateral sclerosis. Med Hypotheses. 1997;48:281-96. 